\title{
Correction to: Automorphisms of the Doubles of Purely Non-Abelian Finite Groups
}

\author{
Marc Keilberg ${ }^{1}$
}

Published online: 31 January 2019

(C) Springer Nature B.V. 2019

\section{Algebr Represent Theor (2015) 18:12671297 https://doi.org/10.1007/s10468-015-9540-0}

The original version of the article unfortunately contained a mistake. Lemma 3.10, Lemma 4.4, and Theorem 9.4 are incorrect.

We let all notation be as in [3]. The following is a correction to [3, Lemma 3.10], which improperly states an equivalence.

Lemma 3.10 Let $G, H$ be groups; $u: \mathbb{k}^{G} \rightarrow \mathbb{k}^{H}$ a morphism of coalgebras, and $v: \mathbb{k} G \rightarrow$ $\mathbb{k} H$ an algebra morphism. Then the following are equivalent.

(1) $u, v$ satisfy

$$
\text { for all } x, g \in G
$$

$$
v(x) \rightarrow u\left(e_{g}\right)=u\left(e_{x g x^{-1}}\right)
$$

(2) $\left(u^{*} v\right)(g) g^{-1} \in C_{\mathbb{k} G}\left(\operatorname{Im}\left(u^{*}\right)\right)$ for all $g \in G$.

(3) $x u^{*}(h) x^{-1}=u^{*}\left(v(x) h v(x)^{-1}\right)$ for all $x \in G$ and $h \in H$.

Any of these imply that $u^{*} v$ is a normal algebra endomorphism of $\mathbb{k} G$.

Proof The equivalence stated here was in fact correctly proven in [3, Lemma 3.10] by simply rewriting the equation. The error there is in the subsequent assertion that the condition $\left(u^{*} v\right)(g) g^{-1} \in C_{\mathbb{k} G}\left(\operatorname{Im}\left(u^{*}\right)\right)$ for all $g \in G$ is equivalent to normality of $u^{*} v$. A counterexample is simple. Let $G=H, v$ the trivial morphism of Hopf algebras, and $u$ the identity morphism. Then $u^{*} v$ is trivial and so normal, but does not satisfy $\left(u^{*} v\right)(g) g^{-1}=g^{-1} \in$ $C_{G}\left(\operatorname{Im}\left(u^{*}\right)\right)=Z(G)$ for all $g \in G$ unless $G$ is abelian.

The online version of the original article can be found at https://doi.org/10.1007/s10468-015-9540-0.

\section{Presented by: Vyjayanthi Chari}

Marc Keilberg

keilberg@usc.edu

1 Faculté des Sciences Mirande, Institut de Mathématiques de Bourgogne, Université de Bourgogne, 9 avenue Alain Savary, BP 47870 21078, Dijon Cedex, France 
So let $u, v$ be as in the statement. By definition $u^{*} v$ is normal if and only if $\left(u^{*} v\right)\left(g^{x}\right)=$ $\left(u^{*} v\right)(g)^{x}$ for all $g, x \in G$. As in [5, Lemma 3.2] (see also [4, Section 3]) $u^{*} v$ is normal if and only if $\left(S u^{*} v\right) *$ id is an algebra morphism, if and only if $\left(\left(S u^{*} v\right) *\right.$ id) $\curlyvee\left(u^{*} v\right)$, if and only if $\left(u^{*} v\right)(g) g^{-1} \in C_{\mathbb{k} G}\left(\operatorname{Im}\left(u^{*} v\right)\right)$ for all $g \in G$. Since we always have $C_{\mathbb{k} G}\left(\operatorname{Im}\left(u^{*}\right)\right) \subseteq$ $C_{\mathbb{k} G}\left(\operatorname{Im}\left(u^{*} v\right)\right)$, it follows that Eq. 1 implies that $u^{*} v$ is normal, as desired.

Example 1 This rules out $(p, u, r, v)=(0,1,0,0)$ as defining an element of $\operatorname{End}(\mathcal{D}(G))$ whenever $G$ is non-abelian, as the corresponding map $e_{g} \# x \mapsto e_{g} \# 1$ is easily seen to be an algebra morphism if and only if $G$ is abelian.

However, $(0,0,0,1)$, as in [3, Example 7.2], remains a well-defined element of $\operatorname{End}(\mathcal{D}(G))$ for all $G$.

This error affects a few other results, but thankfully does so in a fairly nominal fashion.

To see this, we must extend [3, Lemma 4.4] as follows.

Lemma 4.4 Let $G, H$ be groups (not necessarily finite). Let $v: G \rightarrow H$ and $w: H \rightarrow G$ be group homomorphisms. Suppose that $Z(H) \operatorname{Im}(v)=H$ and $Z(G) \operatorname{Im}(w)=G$. Then the following all hold:

(1) $C_{G}(\operatorname{Im}(w))=Z(G)$;

(2) $C_{H}(\operatorname{Im}(v))=Z(H)$;

(3) $v(Z(G)) \subseteq Z(H)$;

(4) $w(Z(H)) \subseteq Z(G)$;

(5) $C_{H}(\operatorname{Im}(v w))=Z(H)$;

(6) $C_{G}(\operatorname{Im}(w v))=Z(G)$;

(7) The following are equivalent:

(a) $\quad w v \in \operatorname{End}(G)$ is normal;

(b) $v w \in \operatorname{End}(H)$ is normal;

(c) $w(h)^{g}=w\left(h^{v(g)}\right)$ for all $g \in G$ and $h \in H$;

(d) $v(g)^{h}=v\left(g^{w(h)}\right)$ for all $g \in G$ and $h \in H$.

When any of these hold we also have $\operatorname{ker}(v) \subseteq Z(G)$ and $\operatorname{ker}(w) \subseteq Z(H)$.

Proof Items 1 to 4 were proven in [3, Lemma 4.4]. The equivalence of Items 7a and 7b was also stated, but there is a minor omission in the proof thereof we will correct.

By Item 3 and the assumption $G=Z(G) \operatorname{Im}(w)$ we have

$$
C_{H}(\operatorname{Im}(v))=C_{H}(v(Z(G) \operatorname{Im}(w)))=C_{H}(v(Z(G)) \operatorname{Im}(v w))=C_{H}(\operatorname{Im}(v w)) .
$$

Thus Item 2 implies Item 5. Similarly, Item 1 and 4 and the assumption $H=Z(H) \operatorname{Im}(v)$ implies Item 6.

The equivalence of Item $7 \mathrm{a}$ and $7 \mathrm{c}$, and of Item $7 \mathrm{~b}$ and $7 \mathrm{~d}$, now follows from Lemma 3.10 and its proof. We can then fill in the omission from the proof given in [3, Lemma 4.4] of the equivalence of Item $7 \mathrm{a}$ and $7 \mathrm{~b}$ by using Item 5 and 6 as follows.

Suppose that Item 7a holds. For any $h \in H$, we may write $h=b v(g)$ for some $b \in Z(H)$ and $g \in G$. Then

$$
(v w)(h) h^{-1}=(v w)(b v(g))(b v(g))^{-1}=\left((v w)(b) b^{-1}\right) \cdot v\left((w v)(g) g^{-1}\right) .
$$


From Item 3 and 4 we have $(v w)(b) b^{-1} \in Z(H)$. By normality of $w v$ and Item 5 we have $(w v)(g) g^{-1} \in C_{G}(\operatorname{Im}(w v))=Z(G)$. Therefore $(v w)(h) h^{-1} \in Z(H) \subseteq C_{H}(\operatorname{Im}(v w))$ (indeed, equality holds by Item 5 ) for all $h \in H$, which implies $v w$ is normal, as desired. The other direction of the equivalence follows by symmetry. The statement about $\operatorname{ker}(v)$ follows from $(w v)(g) g^{-1} \in C_{G}(\operatorname{Im}(w v))=Z(G)$, and similarly for $\operatorname{ker}(w)$.

This completes the proof.

The extended equivalence guarantees that all arguments and statements concerning isomorphisms $\mathcal{D}(G) \rightarrow \mathcal{D}(H)$ (which forces $G \cong H$ by [3, Theorem 3.4]) carry through essentially unaltered, as then $w=u^{*}$ and $v$ satisfy the above lemma by [3, Lemma 3.3 and Theorem 3.4]. Thus the error is only particularly relevant to results about morphisms which are not necessarily isomorphisms.

The principle such relevance is in the proof of [3, Theorem 9.4]. The proof of this result needs the corrected Lemma 3.10 to correctly establish that centralizers are mapped appropriately. There is an additional issue where the proof explicitly requires $|\operatorname{class}(s)|=$ $\left|\operatorname{class}\left(u^{*}(s)\right)\right|$, but does not provide adequate justification or assumptions to ensure this. As such we fully state and prove the corrected version here.

Theorem 9.4 Fix groups $G, H$ and a homomorphism $\psi=(p, u, r, v) \in$ $\operatorname{Hom}(\mathcal{D}(G), \mathcal{D}(H))$. Suppose that all of the following hold:

(1) $\psi$ is flippable;

(2) $Z(G) \operatorname{Im}\left(u^{*}\right)=G$;

(3) $\operatorname{ker}\left(u^{*}\right)$ contains no non-trivial commutators.

Then the induced map $\operatorname{Rep}(\mathcal{D}(H)) \rightarrow \operatorname{Rep}(\mathcal{D}(G))$ is given by

$$
(s, \chi) \mapsto\left(u^{*}(s) P(\chi), r^{*}(s) * \chi \circ v\right) .
$$

Proof Let assumptions and notation be as in the statement. By [3, Lemma 4.2] the assumption that $\psi$ is flippable means we have $A \subseteq Z(G), B \subseteq Z(H)$, and that the components are all morphisms of Hopf algebras. Moreover, flippability of $\psi$ by definition means $\left(p^{*}, v^{*}, r^{*}, u^{*}\right) \in \operatorname{Hom}(\mathcal{D}(H), \mathcal{D}(G))$, so by [3, Corollary 2.3] and Lemma 3.10 we have

$$
v(g)^{h}=v\left(g^{u^{*}(h)}\right)
$$

for all $g \in G$ and $h \in H$.

For the remainder of the proof we fix an arbitrary irreducible representation $(s, \chi)$ of $\mathcal{D}(H)$.

By [3, Lemma 9.2] $(s, \chi)$ is sent to a module supported at $u^{*}(s) P(\chi)$. To obtain the desired result, we need only show that the character of $(s, \chi)$ under $\psi$ is the same as the character for $\left(u^{*}(s) P(\chi), r^{*}(s) *(\chi \circ v)\right)$.

First we must justify that $\chi \circ v$ is a well-defined character of

$$
C_{G}\left(P(\chi) u^{*}(s)\right)=C_{G}\left(u^{*}(s)\right),
$$

or equivalently that $v$ restricts to a group homomorphism $C_{G}\left(u^{*}(s)\right) \rightarrow C_{H}(s)$ for any $s \in H$. By Lemma 3.10 we have $v\left(u^{*}(s)\right) s^{-1} \in C_{G}\left(\operatorname{Im}\left(u^{*}\right)\right)$. The assumption $Z(G) \operatorname{Im}\left(u^{*}\right)=G$ implies $C_{G}\left(\operatorname{Im}\left(u^{*}\right)\right)=Z(G)$. Therefore $C_{H}\left(v\left(u^{*}(s)\right)\right)=C_{H}(s)$. Using this, the assumption that $Z(G) \operatorname{Im}\left(u^{*}\right)=G$, and taking $g=u^{*}(s)$ in Eq. 2 we obtain the desired claim. 
Next, the assumption $Z(G) \operatorname{Im}\left(u^{*}\right)=G$ additionally implies class $\left(u^{*}(s)\right)=$ $u^{*}(\operatorname{class}(s))$ for all $s \in H$. Given this equality we then have $|\operatorname{class}(s)|=\left|\operatorname{class}\left(u^{*}(s)\right)\right|$ for all $s \in H$ if and only if $u^{*}$ is one-to-one on conjugacy classes. This in turn is equivalent to $\operatorname{ker}\left(u^{*}\right)$ containing no non-trivial commutators. So by assumptions $|\operatorname{class}(s)|=$ $\left|\operatorname{class}\left(u^{*}(s)\right)\right|$.

Now given $g \in \operatorname{class}\left(P(\chi) u^{*}(s)\right)=P(\chi) \operatorname{class}\left(u^{*}(s)\right)$ we let $y_{g} \in \operatorname{class}(s)$ be such that $P(\chi) u^{*}\left(y_{g}\right)=g$. Such a value of $y_{g}$ exists and is unique by the preceding remarks. Fixing an element $y_{g}^{\prime} \in H$ such that $s^{y_{g}^{\prime}}=y_{g}$, we define $g^{\prime}=u^{*}\left(y_{g}^{\prime}\right)$. By definition, $\left(P(\chi) u^{*}(s)\right)^{g^{\prime}}=g$.

Now let $\xi$ be the character of the image of $(s, \chi)$ under $\psi$, and let $\beta$ be the character of $\left(u^{*}(s) P(\chi), r^{*}(s) *(\chi \circ v)\right)$. By [3, Lemma 9.1],

$$
\beta\left(e_{g} \# h\right)=r^{*}(s)(h) \chi(v(t))
$$

whenever $g \in \operatorname{class}\left(P(\chi) u^{*}(s)\right)=P(\chi) \operatorname{class}\left(u^{*}(s)\right)$ and $h=t^{g^{\prime}}$ for some $t \in$ $C_{G}\left(P(\chi) u^{*}(s)\right)=C_{G}\left(u^{*}(s)\right)$, with $g^{\prime}$ defined as above; and $\beta\left(e_{g} \# h\right)=0$ otherwise.

On the other hand, by [3, equation (9.6)] we have

$$
\xi\left(e_{g} \# h\right)=r^{*}(s)(h) \chi(\gamma)
$$

whenever $g \in \operatorname{class}\left(P(\chi) u^{*}(s)\right)$, and $v(h)=\gamma^{y_{g}^{\prime}}$ for some $\gamma \in C_{H}(s)$, with $y_{g}^{\prime}$ defined as above; and $\xi\left(e_{g} \# h\right)=0$ otherwise.

So suppose $\beta\left(e_{g} \# h\right) \neq 0$. We then have $v(h)=v\left(t^{g^{\prime}}\right)=v(t)^{\left(v u^{*}\right)\left(y_{g}^{\prime}\right)}$. We have previously noted that $\left(v u^{*}\right)\left(y_{g}^{\prime}\right)\left(y_{g}^{\prime}\right)^{-1} \in C_{H}(\operatorname{Im}(v))$, from which it follows that $v(h)=v(t)^{y_{g}^{\prime}}$. We therefore conclude that $\xi\left(e_{g} \# h\right)=\beta\left(e_{g} \# h\right)$ whenever $\beta\left(e_{g} \# h\right) \neq 0$.

Conversely, if $\xi\left(e_{g} \# h\right) \neq 0$ then $v(h)=\gamma^{y_{g}^{\prime}}$ implies $\left(u^{*} v\right)(h)=u^{*}(\gamma)^{g^{\prime}}$. From Lemma 3.10 and the assumption that $Z(G) \operatorname{Im}\left(u^{*}\right)=G$ we conclude that $\left(u^{*} v\right)(h)=d^{-1} h$ for some $d \in C_{G}\left(\operatorname{Im}\left(u^{*}\right)\right)=Z(G)$. Therefore $h=\left(d u^{*}(\gamma)\right)^{g^{\prime}}$ and $d u^{*}(\gamma) \in C_{G}\left(u^{*}(s)\right)$. We conclude that $\beta\left(e_{g} \# h\right) \neq 0$, and so from the preceding argument we must have that $\beta\left(e_{g} \# h\right)=\xi\left(e_{g} \# h\right)$ for all $g, h \in G$.

This completes the proof.

As was previously mentioned, Lemma 3.10 is used here to properly justify that $v$ restricts to a group homomorphism $C_{G}\left(u^{*}(s)\right) \rightarrow C_{H}(s)$. The original statement and proof also omitted the assumption that $\operatorname{ker}\left(u^{*}\right)$ contains no non-trivial commutator. We can see the necessity of this by simply comparing dimensions:

$$
\begin{gathered}
\operatorname{dim}(s, \chi)=|\operatorname{class}(s)| \chi(1) ; \\
\operatorname{dim}\left(u^{*}(s) P(\chi), r^{*}(s) *(\chi \circ v)\right)=\left|\operatorname{class}\left(u^{*}(s)\right)\right| \chi(1) .
\end{gathered}
$$

The proof given in [3] explicitly relied upon $|\operatorname{class}(s)|=\left|\operatorname{class}\left(u^{*}(s)\right)\right|$, but did not provide logically sound justification for why this would hold without the explicit assumption on $\operatorname{ker}\left(u^{*}\right)$. Observe that from [3, Theorem 3.12] we have $\operatorname{ker}\left(u^{*}\right) \cap H^{\prime}=1$ is always true for isomorphisms, so Theorem 9.4 is applicable to all isomorphisms.

Example 2 While it is known that derived subgroups of finite groups can contain elements which are not commutators [2], it is perhaps not particularly well-known if it is possible for the derived subgroup to contain a subgroup consisting entirely of such elements (other than the identity) which is normal in the parent group. Any such subgroup would necessarily be central. A search with GAP [1] shows that the smallest such example has order 128. In 
particular, $G=$ SmallGroup $(128,731)$ has an element of order 2 in $Z(G) \subset G^{\prime}$ which is also the unique element of $G^{\prime}$ which is not a commutator.

Subsequently, while $\operatorname{ker}\left(u^{*}\right) \cap H^{\prime}=1$ implies $\operatorname{ker}\left(u^{*}\right)$ contains no non-trivial commutator, the converse need not hold.

The remaining consequences of the error in [3, Lemma 3.10] are as follows.

- The statement of [5, Lemma 9.2] must replace the statement " $u^{*} v$ is normal" with Eq. 1, or any equivalent form from Lemma 3.10 .

- Similar changes must be made in the statement of [5, Corollary 9.3].

Publisher's Note Springer Nature remains neutral with regard to jurisdictional claims in published maps and institutional affiliations.

\section{References}

1. GAP. GAP - Groups, Algorithms, and Programming, Version 4.8.4. http://www.gap-system.org (2016)

2. Isaacs, I.M.: Commutators and the commutator subgroup. Amer. Math. Mon. 84(9), 720-722 (1977). ISSN 00029890, 19300972. http://www.jstor.org/stable/2321253

3. Keilberg, M.: Automorphisms of the doubles of purely non-abelian finite groups. Algebras Represent. Theory 18(5), 1267-1297 (2015). ISSN 1386-923X. https://doi.org/10.1007/s10468-015-9540-0

4. Keilberg, M.: Quasitriangular structures of the double of a finite group. Commun. Algebra 0(0), 1-34 (2018). https://doi.org/10.1080/00927872.2018.1461883

5. Keilberg, M., Schauenburg, P.: On tensor factorizations of Hopf algebras. Algebra Number Theory 10(1), 61-87 (2016). https://doi.org/10.2140/ant.2016.10.61 